\title{
PRIMARY GASTROINTESTINAL STROMAL TUMOUR OF PROSTATE: A CASE REPORT OF A RARE TUMOUR
}

\author{
M. Arshad Irshad Khalil, Nouman Khan, Azfar Ali, Khurram Mir \\ Department of Surgical Oncology, Shaukat Khanum Memorial Cancer Hospital and Research Centre, Lahore, \\ Pakistan
}

Received: 30 January 2018 / Accepted: 25 June 2018

\begin{abstract}
A 70-year-old gentleman underwent prostatectomy for bladder outlet obstruction due to enlarged prostate and was found to have primary extragastrointestinal stromal tumour (EGIST). He has been started on imatinib therapy and is presently on follow-up. Prostatic EGIST should be one of the differential diagnoses in patients with enlarged prostate with normal prostate-specific antigen levels.
\end{abstract}

Key words: Prostate, gastrointestinal stromal tumour, PSA

\section{Introduction}

Gastrointestinal stromal tumours (GIST) are common in the digestive tract. They have their origin in the pacemaker cells specific to the GI tract. Since similar cells are known to exist in the prostate, such tumours can very rarely be found to arise from this site also. They can be recognised based on histology using specific immunohistochemical stains and can be treated by immunotherapy, surgery or combination of the two methods. ${ }^{[1,2]}$ In this case report, a primary prostatic extra-GIST (EGIST) is presented, which has been treated with imatinib (IM) and is considered for surgery.

\section{Case Report}

A 70-year-old male patient had undergone transvesical prostatectomy in September 2017, for complaints of severe lower urinary tract symptoms i.e., hesitancy, poor stream and sense of incomplete voiding for the past 5 months. As per patient's record pre-operative examination revealed an enlarged firm nodular prostate. His pre-operative prostate-specific antigen (PSA) level was $2.67 \mathrm{ng} / \mathrm{ml}$.

Histopathologic examination of specimen from revealed features consistent with GIST.

Correspondence: Dr. M. Arshad Irshad Khalil, Department of Surgical Oncology, Shaukat Khanum Memorial Cancer Hospital and Research Centre, Lahore, Pakistan.

Email: arshadkmcite@gmail.com
Immunohistochemical staining showed the following reactivity:

- CD117 diffuse positive.

- DOG-1 diffuse positive.

- CD34 positive.

- Desmin negative.

- ASMA negative.

He was further worked up with colonoscopy and upper GI endoscopy to find any primary tumour in the lower GI tract. No gross abnormality was found on these examinations.

He was accepted in Shaukat Khanum Memorial Cancer Hospital and Research Centre through walk-in clinic and presented to the Department of Uro-oncology. Histopathology was reviewed at our institution as per policy. It showed that on gross examination, there were multiple irregular fragments of greyish-brown tissue, measuring $11.0 \mathrm{~cm} \times 10.0 \mathrm{~cm} \times 5.0 \mathrm{~cm}$. On sectioning, greyish-brown and haemorrhagic tissue was noted. The whole specimen was submitted in three blocks.

Microscopic examination shows neoplastic lesion, which was composed of fascicles of spindle cells. These neoplastic cells contain a moderate amount of eosinophilic cytoplasm with oval-to-elongated nuclei and inconspicuous nucleoli. At places, cytoplasmic vacuolisation is also appreciable. The mitotic count was 2-3/50 high-power fields (HPF) [Figure 1]. No normal prostatic tissue was seen. 
Immunohistochemical staining with CD117 and DOG1 and the specimen showed strong positivity. CD117 showed diffuse cytoplasmic staining in tumour. DOG1 showed diffuse cytoplasmic and membranous staining in tumour cells. Details of immunohistochemical staining are as follows:

- DOG1: Clone (SP31).

- Performed on Ventana BenchMark XT.

- CD117: Clone T595.

- $\quad$ Performed on Bond Leica 3.

His chest and abdominal computed tomography (CT) was also reviewed which was unremarkable. Review of pelvic magnetic resonance imaging (MRI) [Figure 2] scan showed a significantly enlarge residual prostate, but no abnormality could be identified in the rectum. Furthermore, the prostate was completely separated from the rectum excluding the possibility of a rectal GIST that may be involving the prostate.

Pathological diagnosis was GIST based on the histology and immunostaining. Since no other primary site could be identified on endoscopy and imaging, it was concluded to be a case of primary prostatic EGIST, and as on histopathology, there are no differences between GISTs from GI or prostatic origins.

The case was presented in the Multidisciplinary Team Meeting and it was discussed that, based on the large size of the residual prostate and its proximity to the rectum, complete surgical excision may not be possible, and there is a risk of rectal injury. The treatment plan was discussed

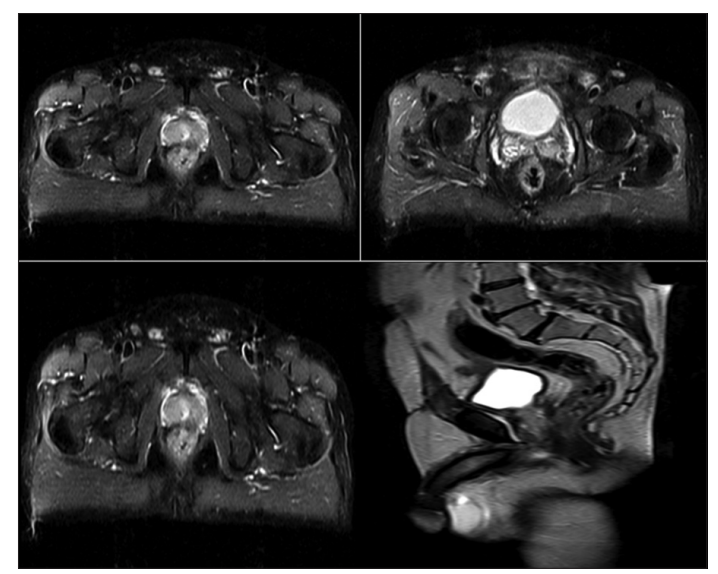

Figure 1: Magnetic resonance imaging (T2W sequence): Significant residual prostate is noted with the patient and he was referred to the medical oncologist and started on imitanib mesylate, a selective protein tyrosine kinase inhibitor at the dose of $400 \mathrm{mg}$ once daily to see the response. Follow-up CT [Figure 3] scan has been performed before the commencement of IM therapy which showed enlarged prostate without contiguous visceral involvement and local or distant metastasis.

The patient has taken imitanib mesylate for almost 6 months now. Repeat MRI pelvis done on 29 May 2018 showed a small focus of nodular enhancement interposed

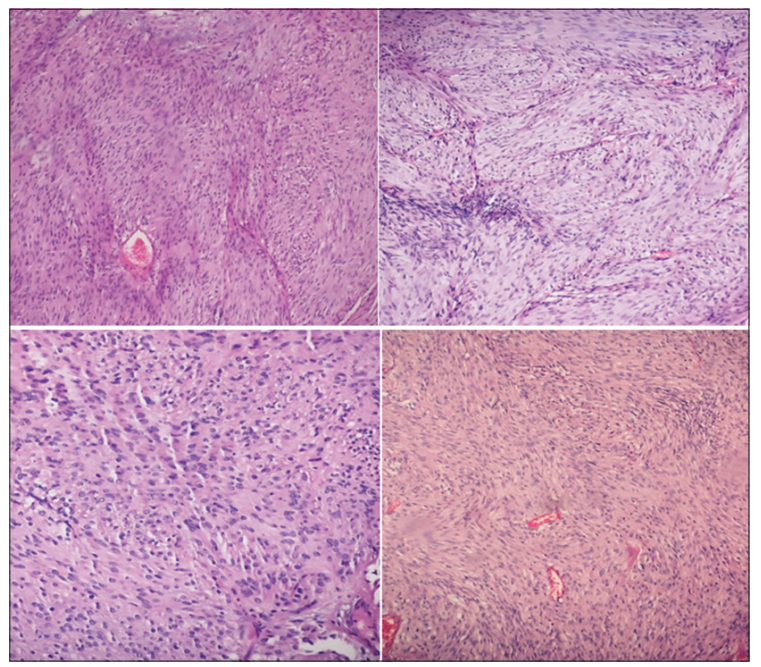

Figure 2: Biopsy $(\times 40$ images $)$ shows a collection of bland spindle cells in the form of short intersecting fascicles showing eosinophilic fibrillar cytoplasm. Perinuclear cytoplasmic vacuoles and minimal pleomorphism are seen

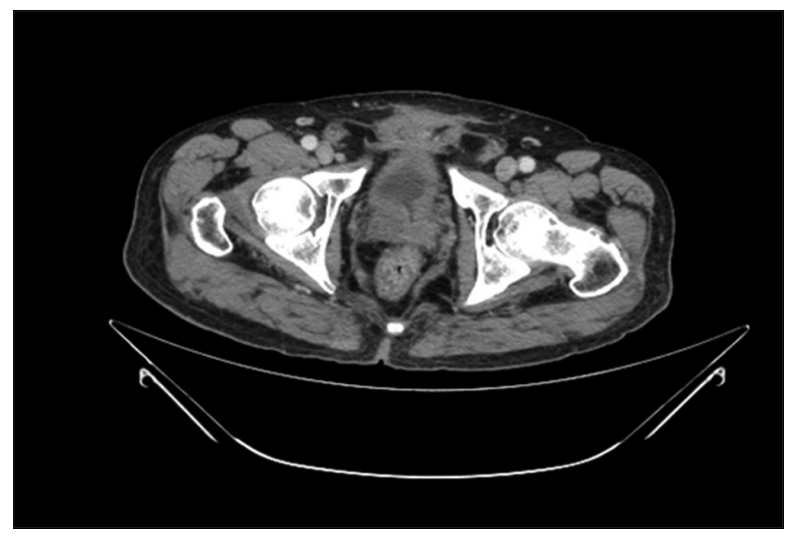

Figure 3: Computerised tomographic scan showing enlarge prostate without any local visceral or nodal involvement 
between the lower rectums and the prostate is suggestive of residual tumour [Figure 4]. The case was again discussed in multidisciplinary team, and it was noted that as there has been no response to imitanib mesylate over the period and the risk of incomplete resection and rectal injury remains, it will be discussed with GI surgeon for combined radical prostatectomy and proctectomy with temporary or permanent colostomy. In the meanwhile, the dose of imitanib mesylate has been increased to $600 \mathrm{mg}$ a day.

\section{Discussion}

GIST is a tumour of mesenchymal origin from the GI tract. The EGISTs have similarities with their GI counterparts in their histological and immunohistochemical characteristics, but they have little or no connection with

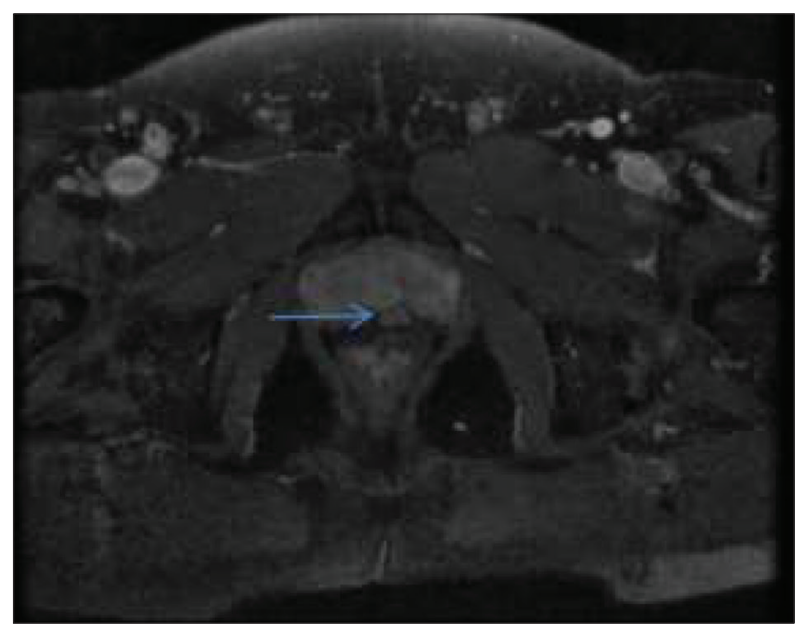

Figure 4: Follow-up magnetic resonance imaging shows a new contrast-enhancing nodule (arrow) the adjacent serosal surfaces of the GI tract. These EGISTs constitute $5-10 \%$ of such stromal tumours and patients have a mean age of 58 years. ${ }^{[3-7]}$

Van Der Aa et al. reported the first case of prostatic EGIST. ${ }^{[5]}$ Prostate is also the very rare site of the origin of other mesenchymal malignancies. These include sarcomas which account for approximately $0.1 \%-0.2 \%$, including leiomyosarcomas and rhabdomyosarcomas.

Other primary sarcomas are extremely uncommon, which also include primary prostatic EGIST along with malignant fibrous histiocytoma, angiosarcoma, osteosarcoma, chondrosarcoma, malignant peripheral nerve sheath tumour and synovial sarcoma. ${ }^{[9]}$ Due to the proximity of prostate with the rectum, the possibility of patients presenting with urinary symptoms from large GIST of rectal origin (due to large volume causing mass effect) should be excluded.

Table 1 presents the previously reported cases of EGIST including the present case. Patients commonly present with burning micturition, urinary frequency, haematuria, and pelvic or perineal pain. They mostly remain clinically silent in the initial stages and attain large size. Physical examination and imaging often reveal a significantly enlarged prostate. PSA level is almost always within the normal range 1. If resectable, surgery is the definitive treatment option in patients with prostatic EGISTs. ${ }^{[10,11]}$

If possible, complete en bloc removal of the tumour and the surrounding organs that are involved should be performed. Conventional chemotherapy and radiotherapy are not effective in the treatment of EGISTs. IM, which is

Table 1: Reported cases of prostatic extra gastrointestinal stromal tumours

\begin{tabular}{|c|c|c|c|}
\hline Reference & Age (years) & T-size $(\mathrm{cm})$ & Treatment \\
\hline Van der Aa et al., $2005^{[5]}$ & 49 & 14.2 & Imatinib \\
\hline Lee et $a l ., 2006^{[4]}$ & 75 & 6.7 & TURP+RP \\
\hline Yinghao et al., 2007 ${ }^{[10]}$ & 49 & 8 & $\mathrm{RP}$ \\
\hline Ou et al., $2013^{[9]}$ & 39 & 10 & RP+Imatinib \\
\hline Zhang et al., 2014 ${ }^{[3]}$ & 31 & 6.5 & Imatinib \\
\hline Liu et al., 2014 & 55 & 10.5 & $\mathrm{RP}+$ Imatinib \\
\hline Etit et al., $2017^{[8]}$ & 56 & 6 & Enucleation \\
\hline Present case & 70 & 5 & Imatinib \\
\hline
\end{tabular}

TURP: Transurethral resection of prostate, RP: Radical prostatectomy 
Table 2: Proposed risk factors for aggressiveness in gastrointestinal stromal tumours

\begin{tabular}{l|c|c}
\hline Risk of aggressiveness & Size (largest diameter) $\mathbf{( c m})$ & Mitotic count (/50 HPF) \\
\hline Very low risk & $<2$ & $<5$ \\
\hline Low risk & $2-5$ & $<5$ \\
\hline Intermediate & $<5$ & $6-10$ \\
\hline Risk & $5-10$ & $<5$ \\
\hline High risk & $>5$ & $>5$ \\
\hline & $>10$ & Any mitotic rate
\end{tabular}

HPF: High-power fields

a tyrosine kinase inhibitor, is used as methods of adjuvant therapy. It also has a role in the treatment of advanced, unresectable, and metastatic GIST. Fletcher et al. proposed the first risk classification system for GIST in 2002, which is termed as 'NIH classification'. Its goal was to develop a consensus approach to diagnose and help in morphologic prognostication of GISTs. The criteria so developed were based on tumour size and mitotic count [Table 2]. Similar predictive value was also proposed by Reith et al., for EGISTs in his analysis of 48 patients, according to whom mitotic activity and necrosis have independent predictive value. ${ }^{[7]}$

Several other workers evaluated the NIH classification and proposed subsequent prognostic schemes for GISTs, including other variables such as the anatomic site, because prognosis of GISTs from gastric and non-gastric origins was noted to be different. The other two widely used classification systems include the NCCN risk classification for GIST, based on the work of Miettinen and Lasota in 2006, ${ }^{[12]}$ and the American Joint Committee on Cancer prognostic scheme.

Due to the scarcity of data on the primary prostatic EGISTs, the implementation of classification schemes for GISTs on these tumours has not been validated. In the present case, the relatively small size $(5 \mathrm{~cm})$, low mitotic counts (2-3/50 HPFs) and absence of necrosis were the low features. Therefore, it was decided to go for IM therapy and keep the patient on follow-up.

Although GISTs exhibit a variable biological behaviour, $10-30 \%$ of them are malignant. ${ }^{[13]}$ This warrants requirement of a close follow-up based individually on tumour characters. The higher the risk, early is the chance of recurrence with median time of 2 years after surgery. Due to paucity of data, it is difficult to predict the malignant potential of prostatic EGISTs; however, it seems to be quite similar to that of intestinal GISTS. Tailored follow-up schedule using appropriate imaging is, therefore, required.

\section{Conclusion}

In this case report, we present one of the rarest prostatic malignancies. We recommend that:

1. EGIST should be considered in the differential diagnosis of the large firm to hard feeling prostates with normal PSA values.

2. Surgical resection should be considered in all cases where possible.

3. If not then, IM represents the alternative therapy and can also be used in combination with surgery.

4. Long-term follow-up is required in all cases.

\section{Conflict of Interest}

The authors declare that they have no conflict of interest.

\section{References}

1. Liu S, Yu Q, Han W, et al. Primary gastrointestinal stromal tumor of the prostate: A case report and literature review. Oncol Lett 2014;7:1925-9.

2. Appelman HD. Morphology of gastrointestinal stromal tumors: Historical perspectives. J Surg Oncol 2011; 104:874-81.

3. Zhang ZH, Feng GW, Liu ZF, et al. A young man with primary prostatic extra-gastrointestinal stromal tumor: A rare case report and review of the literature. Int J Clin Exp Pathol 2014;7:1764-70.

4. Lee $\mathrm{CH}$, Lin $\mathrm{YH}$, Lin HY, et al. Gastrointestinal stromal tumor of the prostate: A case report and literature review. Hum Pathol 2006;37:1361-5.

5. Van der Aa F, Sciot R, Blyweert W, et al. Gastrointestinal stromal tumor of the prostate. Urology 2005;65:388. 
6. Miettinen M, Sobin LH, Lasota J. Gastrointestinal stromal tumors presenting as omental masses-a clinicopathologic analysis of 95 cases. Am J Surg Pathol 2009;33:1267-75.

7. Reith JD, Goldblum JR, Lyles RH, et al. Extragastrointestinal (soft tissue) stromal tumors: An analysis of 48 cases with emphasis on histologic predictors of outcome. Mod Pathol 2000;13:577-85.

8. Etit D, Kar H, Ekinci N, et al. Extra-gastrointestinal stromal tumor of prostate. Balkan Med J 2017;34:168-71.

9. $\mathrm{Ou}$ Z, Cao Z, He Y, et al. Diagnosis and multimodal therapy for extragastrointestinal stromal tumor of the prostate: A case report. Exp Ther Med 2013;6:378-80.

10. Yinghao S, Bo Y, Xiaofeng G. Extragastrointestinal stromal tumor possibly originating from the prostate. Int J Urol 2007;14:869-71.

11. Yamamoto H, Oda Y, Kawaguchi $\mathrm{K}$, et al. C-kit and PDGFRA mutations in extragastrointestinal stromal tumor (gastrointestinal stromal tumor of the soft tissue). Am J Surg Pathol 2004;28:479-88.

12. Miettinen M, Lasota J. Gastrointestinal stromal tumors: Pathology and prognosis at different sites. Semin Diagn Pathol 2006;23:70-83.

13. Miettinen M, Sobin LH, Lasota J. Gastrointestinal stromal tumors of the stomach: A clinicopathologic, immunohistochemical, and molecular genetic study of 1765 cases with long-term follow-up. Am J Surg Pathol 2005;29:52-68. 